\title{
Les coûts du diabète sur 10 ans au Canada : intégration des coûts en soins de santé imputables au diabète à un modèle de prédiction de son incidence
}

\author{
Anja Bilandzic, M. Sc. (1); Laura Rosella, Ph. D (1, 2, 3)
}

Cet article a fait l'objet d'une évaluation par les pairs.

\section{Résumé}

Introduction : Notre objectif était d'estimer les coûts de santé directs liés au traitement du diabète au Canada sur 10 ans, à l'aide de données de sondages nationaux et d'un outil de prévision du risque de diabète, ainsi que les coûts par personne.

Méthodologie : Nous avons utilisé le Diabetes Population Risk Tool pour estimer le nombre de nouveaux cas de diabète chez les personnes âgées de 20 ans et plus sur 10 ans (jusqu'en 2022) à l'aide des données de 2011 et de 2012 de l'Enquête sur la santé dans les collectivités canadiennes. Nous avons évalué les coûts liés au diabète à partir d'une étude de cohorte faisant appel à l'appariement par score de propension, en utilisant la base de données sur le diabète de l'Ontario ainsi que d'autres données administratives. Nous avons calculé les coûts totaux en utilisant les coûts associés aux nouveaux cas, en tenant compte du sexe, de l'année du diagnostic et des taux de mortalité annuels dus à la maladie.

Résultats : D'après nos calculs, le risque de développer le diabète sur 10 ans s'élevait pour la population canadienne en 2011-2012 à 9,98 \%, soit 2,16 millions de nouveaux cas. Les coûts totaux en soins de santé imputables au diabète pendant cette période sont de 15,36 milliards (7,55 milliards pour les femmes et 7,81 milliards pour les hommes). Ce sont les hospitalisations de courte durée qui constituent la majeure partie de ces coûts $(43,2 \%)$. Des interventions menées au sein de la population entraînant une perte de poids de $5 \%$ au sein de la population permettraient de réduire les coûts des soins de santé de 2,03 milliards de dollars. Une réduction du risque de $30 \%$ obtenue grâce à des interventions auprès des personnes à plus haut risque de développer le diabète (c.-à-d. les $10 \%$ au sommet du groupe à risque le plus élevé) entrânerait des économies de l'ordre de 1,48 million de dollars.

Conclusion : D'ici 2022, le diabète va constituer un lourd fardeau financier pour le système de santé canadien. Notre méthode de calcul des coûts à venir offre aux décideurs et aux planificateurs un outil accessible et clair susceptible de leur permettre de prévoir les dépenses imputables à la maladie et les économies potentielles de coûts associées aux interventions.

Mots-clés : diabète, économies, coûts attribuables, modèle de prédiction, incidence, Canada

\section{Introduction}

La prise en charge et la prévention du diabète demeurent une priorité en matière de santé au Canada. Comme approximativement 1,96 million de personnes vivent avec le diabète ${ }^{1}$ et qu'on s'attend à une augmentation du nombre de personnes qui vont développer cette maladie chronique, il est important d'envisager des stratégies à grande échelle permettant de freiner la maladie. En outre, le diabète représente une contrainte importante pour le système

\section{Points saillants}

- Nous avons créé un outil accessible et clair visant à aider les décideurs du domaine de la santé à calculer les coûts associés au diabète.

- Nous avons calculé le nombre de nouveaux cas chez les personnes âgées de 20 ans et plus pour 10 ans au Canada (2011-2012 à 2021-2022) et nous avons lié ces résultats aux coûts individuels réels en soins de santé imputables au diabète.

- D'ici 2022, on s'attend à ce que 2,16 millions de nouveaux cas de diabète soient diagnostiqués, lesquels entraîneront des coûts en soins de santé atteignant 15,36 milliards de dollars.

- Cet outil permet de modéliser au sein de la population différentes interventions de réduction du risque : par exemple, une diminution de poids de $5 \%$ au sein de la population permettrait de réduire les coûts de 2,03 milliards de dollars et une diminution du risque de $30 \%$ au sein du groupe à plus haut risque entraînerait des économies de l'ordre de 1,48 milliard de dollars.

de santé canadien. On estime en effet qu'en 2008, les coûts des soins hospitaliers, des services médicaux et des médicaments pour le diabète ont atteint 2,18 milliards $^{2}$ de dollars. En se fondant sur les 3,7 millions de cas prévalents calculés à l'aide d'un modèle de coûts spécifiquement conçu pour le diabète ${ }^{3}$, l'Association canadienne du diabète a prévu que les coûts directement liés au diabète vont être 
de 3,1 milliards de dollars en 2020. À l'échelle individuelle, Goeree et ses collaborateurs ont estimé que les coûts par nouveau cas de diabète en Ontario sont d'approximativement 2930 \$ au cours de la première année suivant le diagnostic et de 1240 \$ les années suivantes ${ }^{4}$. Plus récemment, Rosella et ses collaborateurs, en élargissant la portée de ces travaux pour $\mathrm{y}$ inclure un plus grand nombre de frais directs pour la province, ont établi un coût moyen imputable au diabèe à 9731 \$ pour les femmes et à 10315 \$ pour les hommes pour huit ans de suivi ${ }^{5}$.

Malgré l'existence de travaux au Canada visant à évaluer les coûts futurs du diabète $^{3,6}$, la plupart des estimations et des modèles sont complexes, manquent de clarté et ne sont pas facilement utilisables par les décideurs. Un outil permettant aux décideurs de réaliser une estimation de l'impact économique des nouveaux cas de diabète sur le système de santé entraînerait une planification plus efficace en prévention du diabète. Pouvoir quantifier l'impact des mesures actuelles sur l'apparition des nouveaux cas et sur les futures dépenses en santé associées constitue un avantage considérable dans l'évaluation des stratégies lors de la mise en œuvre d'un programme. L'objectif de cette étude est dans un premier temps d'estimer, à partir des données d'un sondage national et des coûts par personne, les coûts en matière de santé directement liés aux nouveaux cas de diabète sur 10 ans au Canada et d'inscrire ces coûts dans le contexte d'un outil de prédiction du risque. Il s'agit en second lieu d'appliquer cet outil à deux scénarios d'intervention visant à assurer la diminution de l'incidence du diabète au sein de la population.

\section{Méthodologie}

\section{Risque de diabète et incidence}

Pour faire une estimation du risque et du nombre de nouveaux cas de diabète sur 10 ans, nous avons utilisé la version $2.0 \mathrm{du}$ Diabetes Population Risk Tool. Le DPoRT 2.0 est une mise à jour du DPoRT, un algorithme de prédiction mis au point pour calculer les risques à venir au sein de la population et le nombre de nouveaux cas diagnostiqués par les médecins chez les adultes de 20 ans et plus. Le DPoRT repose sur les données d'un sondage national jumelées individuellement à un registre de cas de diabète validés au moyen des dossiers des patients. Cette cohorte a ensuite servi à créer des modèles de survie selon le sexe à l'aide de facteurs de risque de référence tirés de l'enquête sur l'incidence du diabète. Nous avons plus spécifiquement évalué la probabilité de diagnostic de diabète par les médecins entre la date de l'entrevue et celle de clôture des données (pour cause de décès ou de fin de suivi). Le modèle a été développé pour une cohorte de l'Ontario et ses prédictions ont été validées avec l'incidence réellement observée dans deux autres cohortes, l'une en Ontario et l'autre au Manitoba. Les variables utilisées dans ces deux modèles tenant compte du sexe combinaient des données sur l'hypertension, l'origine ethnique, le niveau de scolarité, le statut vis-à-vis de l'immigration, l'indice de masse corporelle, le tabagisme, les maladies coronariennes et le revenu. Les détails complets sur les spécifications et la validation du modèle peuvent être consultés ailleurs $^{7}$. Le modèle de régression, applicable à toutes les enquêtes de population en santé menées à l'échelle nationale, a fait l'objet d'une mise à jour (DPoRT 2.0) et d'une application aux cibles de prévention du diabète ${ }^{8}$.

Nous avons utilisé pour cette étude le DPoRT 2.0 afin de générer des prévisions de l'incidence à partir de l'Enquête sur la santé dans les collectivités canadiennes (ESCC) menée en 2011 et 2012. L’ESCC permet de recueillir des renseignements sur les caractéristiques individuelles, l'état de santé et les déterminants de la santé de la population canadienne. Il s'agit d'une enquête représentative à l'échelle nationale, de conception transversale, menée de manière continue et dont un rapport est produit chaque année. Elle couvre $98 \%$ de la population de 12 ans et plus et en sont exclus les résidents des réserves et des terres de la Couronne, la population vivant en établissement, les membres à temps plein des Forces canadiennes et les personnes vivant dans certaines régions éloignées ${ }^{9}$. La taille de l'échantillon est passée de 124929 personnes à 90631 personnes après application des facteurs d'exclusion (dont les répondants âgés de moins de 20 ans et ceux déjà atteints de diabète), représentant au final 21598180 personnes après pondération.

\section{Scénarios d'intervention}

Outre les estimations de référence (caractéristiques individuelles et facteurs de risque mentionnés plus haut), nous avons testé deux scénarios afin d'examiner quelle influence aurait la mise en œuvre d'interventions visant à réduire le risque de diabète sur l'incidence de la maladie et sur les coûts du système de santé.

Nous avons d'abord modélisé une intervention non ciblée entraînant une perte de poids de $5 \%$ au sein de la population. Cette diminution aurait un effet positif sur la glycémie et sur la santé cardiovasculaire d'un point de vue clinique ${ }^{10}$ et constituerait une perte de poids modeste et réaliste pour de nombreuses personnes. Cette intervention pourrait être due à un changement à grande échelle, par exemple une modification de l'environnement bâti (il a été démontré que les taux de prévalence de surpoids et d'obésité des populations vivant dans des secteurs favorables à la marche sont moins élevés ${ }^{11}$ ) ou une amélioration de l'étiquetage nutritionnel.

Nous avons ensuite mis en place un scénario d'intervention dans le cadre duquel les personnes du plus haut décile (c.-à-d. ceux qui présentent un risque de développer le diabète en 10 ans de 22,6\% ou plus) ont été ciblées pour une intervention menant à une réduction de $30 \%$ de leur risque. Ce type d'approche correspond par exemple à un programme d'intervention ciblant les modes de vie ou à une intervention pharmacologique dont l'efficacité a été démontrée lors d'essais aléatoires ${ }^{12}$.

\section{Estimation des coûts imputables au diabète}

Pour estimer les coûts futurs imputables au diabète, nous avons utilisé les résultats d'une récente étude de cohorte faisant appel à l'appariement par score de propension ${ }^{5}$. Pour résumer, on a utilisé dans le cadre de cette étude la Base de données sur le diabète de l'Ontario (ODD) pour cerner les nouveaux cas de diabète diagnostiqués par des médecins entre le $1^{\text {er }}$ avril 2004 et le 31 mars 2012. Trois sujets témoins non diabétiques ont été jumelés à chaque personne diabétique puis ont été appariés en fonction d'une date de référence ( \pm 30 jours), de l'âge ( \pm 90 jours) et du logit du score de propension. Ce dernier représentait la probabilité de développer le diabète ou non en fonction d'une régression logistique portant sur l'âge, le milieu (rural ou urbain), la comorbidité, la zone géographique et le quintile de revenu du voisinage comme variables de prédiction. 
Les soins de santé individuels directs ont été recensés annuellement pendant les huit années de ce suivi. Leur coût a été évalué en reliant diverses bases de données sur l'utilisation des soins de santé et selon une méthode d'estimation des coûts par personne développée et validée spécifiquement pour les bases de données administratives de l'Ontario ${ }^{13}$. Ces coûts relèvent du système de santé et englobent les coûts des hospitalisations, les visites aux services d'urgence, les chirurgies ambulatoires, la dialyse, les visites aux cliniques d'oncologie, les soins médicaux et non médicaux rémunérés à l'acte et les soins médicaux non rémunérés à l'acte, les médicaments d'ordonnance, les frais de laboratoire, la réadaptation, les soins continus complexes, les soins de longue durée, les hospitalisations en santé mentale, les soins à domicile et les appareils médicaux. Les coûts imputables au diabète ont été calculés en faisant la différence entre les coûts concernant les personnes avec diabète et ceux des personnes sans diabète.

\section{Calcul des coûts}

Nous avons mis au point un calculateur de coûts utilisant les prédictions de l'incidence du DPoRT 2.0 et les valeurs des coûts par patient afin d'estimer les coûts en soins de santé liés au diabète sur 10 ans. Tous les calculs ont été effectués en tenant compte du sexe, afin de refléter les différences dans l'utilisation des services de santés, voire en matière de soins autoadministrés ${ }^{14}$. Le nombre estimé de nouveaux cas par année a été multiplié par les coûts annuels par patient correspondants, en fonction du temps écoulé depuis le diagnostic du diabète et en tenant compte des taux de mortalité annuels, lesquels ont été générés à partir des taux de mortalité selon l'âge de l'ODD correspondant à l'année du suivi. Nous avons supposé que les décès se produisaient au milieu de l'année et nous avons par conséquent estimé pour l'année du décès la moitié des coûts engendrés par une personne. Les estimations des coûts individuels étant fondées sur les huit années de suivi de l'analyse, nous avons supposé que les coûts associés aux personnes ayant engendré des frais pendant les $9^{\mathrm{e}}$ et $10^{\mathrm{e}}$ années suivant le diagnostic avaient la même valeur pécuniaire que ceux de la $8^{\text {e }}$ année. Comme on observe une tendance à la baisse des coûts en soins de santé au cours des huit premières années, nous avons mené une analyse de sensibilité selon laquelle les coûts des $9^{\mathrm{e}}$ et $10^{\mathrm{e}}$ années étaient estimés suivant une tendance linéaire, afin de voir l'effet du changement des coûts individuels sur le total des coûts estimés.

\section{Distribution des coûts par secteur}

Afin d'estimer la charge des coûts par secteur, nous avons converti en pourcentages la moyenne des coûts par segment de soins de santé pendant les huit années de suivi et nous l'avons multipliée par les coûts totaux estimés par le calculateur de coûts.

Nous avons réalisé toutes les analyses statistiques à l'aide du logiciel SAS, version 9.4 (SAS Institute Inc., Cary, Caroline du Nord, États-Unis).

\section{Résultats}

Le risque estimé sur 10 ans de développer le diabète pour l'ensemble de la population canadienne est de $9,98 \%$, soit 2156000 nouveaux cas entre 2011-2012 et 2021-2022. Le risque est plus élevé chez les hommes que chez les femmes (11,23\% contre 8,85\%), avec dans l'ensemble un plus grand nombre de nouveaux cas chez les hommes. Le coût estimé total des soins de santé engendrés par ces nouveaux cas s'élève à 15,36 milliards de dollars.
Si une intervention auprès de la population de faible impact et de large portée était mise en place et qu'elle entraînait une perte moyenne de poids de $5 \%$ au sein de la population, le risque estimé de développer le diabète en 10 ans passerait à 8,67 \%, soit 1873000 cas au cours de la période (tableau 1). Ce nombre réduit de nouveaux cas entraînerait des coûts de 13,33 milliards de dollars, représentant une économie de 2,03 milliards de dollars par rapport aux caractéristiques de référence.

En revanche, si une intervention ciblant les personnes à plus haut risque de développer le diabète (soit les $10 \%$ au sommet du groupe à risque le plus élevé) était menée, le risque global de développer le diabète serait de $9,02 \%$, ce qui correspond à 1949000 nouveaux cas et à un coût total de 13,88 milliards de dollars (tableau 1). Une économie de 1,48 milliard de dollars serait réalisée par rapport au scénario de référence.

Avec une estimation des coûts des $9^{\text {e }}$ et $10^{\mathrm{e}}$ années à l'aide d'une tendance linéaire fondée sur l'observation de la $1^{\text {re }}$ à la $8^{\text {e }}$ année, les résultats ne diffèrent pas beaucoup des estimations avec coûts équivalents pendant les $8^{\mathrm{e}}$, $9^{\mathrm{e}}$ et $10^{\mathrm{e}}$ années. Cette différence représentant approximativement 15,96 millions de dollars, nous

TABLEAU 1

Coûts des soins de santé imputables au diabète, scénario de référence et deux scénarios hypothétiques d'intervention, Canada, hommes et femmes, 2011-2012 à 2021-2022

\begin{tabular}{|c|c|c|c|c|}
\hline & & $\begin{array}{l}\text { Risque }^{\mathrm{a}} \text { sur } \\
10 \text { ans (\%) }\end{array}$ & $\begin{array}{l}\text { Incidence (nombre } \\
\text { de cas, en milliers) }\end{array}$ & $\begin{array}{l}\text { Coûts globaux sur } \\
10 \text { ans (milliards de \$) }\end{array}$ \\
\hline \multicolumn{5}{|c|}{ Caractéristiques de référence } \\
\hline \multicolumn{2}{|c|}{ Ensemble } & 9,98 & 2156 & 15,36 \\
\hline \multirow{2}{*}{ Sexe } & Femmes & 8,85 & 1000 & 7,55 \\
\hline & Hommes & 11,23 & 1156 & 7,81 \\
\hline \multicolumn{5}{|c|}{ Perte de poids de $5 \%$ au sein de la population } \\
\hline \multicolumn{2}{|c|}{ Ensemble } & 8,67 & 1873 & 13,33 \\
\hline \multirow{2}{*}{ Sexe } & Femmes & 7,79 & 880 & 6,64 \\
\hline & Hommes & 9,64 & 993 & 6,70 \\
\hline \multicolumn{5}{|c|}{ Réduction du risque de $30 \%$ dans le groupe à risque le plus élevéb } \\
\hline \multicolumn{2}{|c|}{ Ensemble } & 9,02 & 1949 & 13,88 \\
\hline \multirow{2}{*}{ Sexe } & Femmes & 8,20 & 927 & 6,97 \\
\hline & Hommes & 9,93 & 1022 & 6,91 \\
\hline
\end{tabular}

Abréviation : \$, dollars canadiens.

${ }^{a}$ Risque de diabète dans les 10 ans.

${ }^{\mathrm{b}}$ Groupe présentant le risque le plus élevé de diabète dans les 10 ans, soit 22,6 \% ou plus. 
avons jugé l'utilisation de la méthode de calcul des coûts initialement proposée comme appropriée.

En matière de distribution des coûts, la majeure partie des dépenses en soins de santé est consacrée aux hospitalisations à court terme, soit approximativement 43,2\% (6,64 milliards de dollars). Les coûts rattachés aux médecins, soit 21,9\% (3,37 milliards de dollars), occupent le deuxième rang de l'ensemble des coûts. Les médicaments d'ordonnance et les dispositifs d'assistance comptent pour 16,9 \% des coûts (2,60 milliards de dollars) et ils sont suivis des soins à domicile, des soins non médicaux et des soins de longue durée (1,05 milliard de dollars), puis des services ambulatoires (0,88 milliard de dollars) et enfin des services d'urgence, des chirurgies ambulatoires et des services de consultation (0,83 milliard de dollars) (figure 1$)$.

\section{Analyse}

Entre 2011-2012 et 2021-2022, on prévoit que les nouveaux cas de diabète entraîneront des dépenses en soins de santé de l'ordre de 15,36 milliards de dollars au Canada, et que près des deux tiers seront consacrés aux hospitalisations de courte durée et aux coûts rattachés aux médecins $(65,1 \%)$. Notre étude offre un nouveau moyen d'estimer les futurs coûts en soins de santé imputables aux nouveaux cas de diabète. Le jumelage entre le modèle de prédiction de l'incidence et les coûts imputables à l'échelle individuelle permet d'obtenir des estimations pour différents segments de la population, par exemple selon le sexe et selon les coûts par région. La capacité de prédire le nombre de nouveaux cas annuellement permet également aux utilisateurs de calculer les futurs coûts par an pour n'importe quel nombre d'années de suivi (entre un et dix).

Comme il s'agit d'une nouvelle méthode de calcul qui porte surtout sur l'apparition de nouveaux cas de diabète, il est difficile de comparer ces estimations aux coûts projetés antérieurement. Les estimations canadiennes antérieures sont fondées sur des coûts de santé associés au diabète variés et soit ont porté sur les coûts projetés annuellement en fonction des cas prévalents ${ }^{3,6}$, soit se sont rétrospectivement rapportés aux cas déjà produits ${ }^{15-17}$. Le rapport Le fardeau économique de la maladie au Canada, 2005-2008 (FEMC) offre des estimations globales des coûts imputables à différentes maladies, dont le diabète ${ }^{2}$. Notre méthodologie de calcul des coûts diffère de celle utilisée dans le FEMC, en ce sens que celui-ci réfère à des coûts fondés sur la prévalence alors que nous avons utilisé des coûts fondés sur l'incidence. De plus, nous avons évalué les coûts directement imputables au diabète, alors qu'on ne tient compte dans le FEMC que des coûts globaux de maladie, et ce, en utilisant une cohorte avec appariement par score de propension $^{5}$. Enfin, on ne procède pas dans le FEMC au jumelage des estimations avec la

FIGURE 1

Distribution des coûts directs en soins de santé imputables au diabète (en milliards de dollars) en 10 ans, Canada, 2011-2012 à 2021-2022

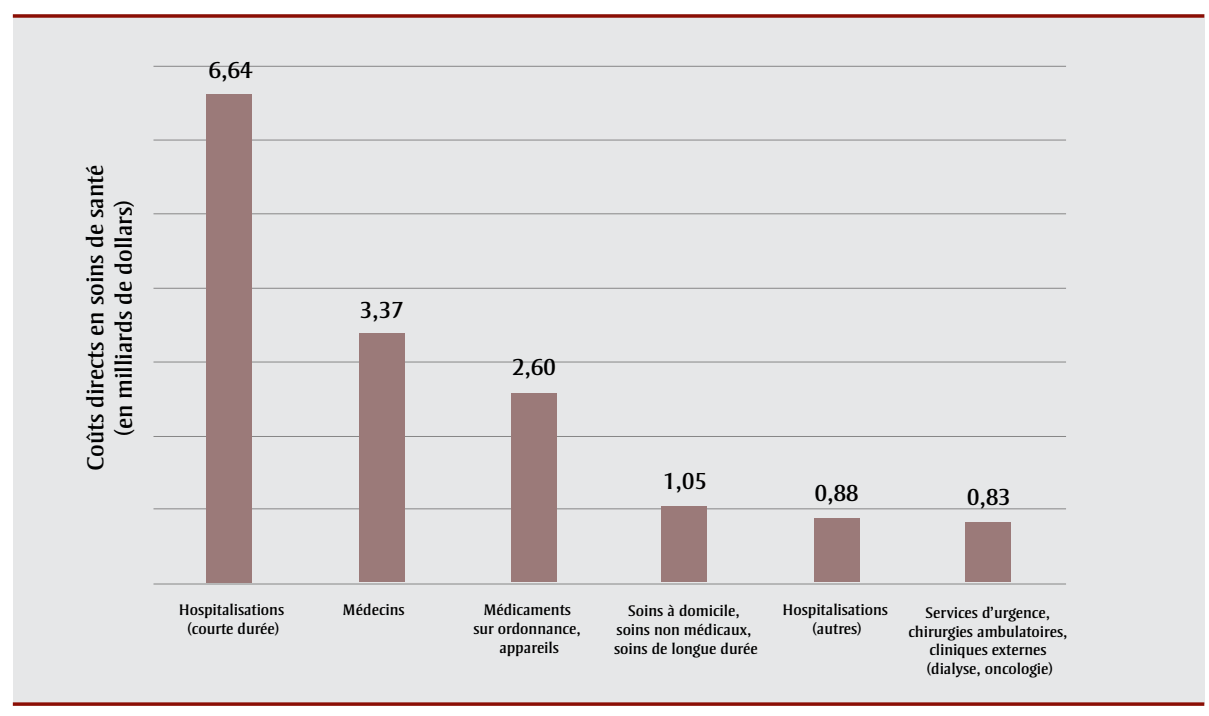

Remarque : Les nombres ont été arrondis.

prédiction des futurs cas et cela ne permet donc ni la planification ni l'évaluation d'interventions en fonction du fardeau des coûts à venir.

\section{Forces et limites}

Notre méthodologie comporte plusieurs points forts remarquables. En premier lieu, les coûts proviennent de données relatives aux coûts en soins de santé réellement observés lors de l'étude d'une cohorte prospective pendant huit ans. Par conséquent, il ne s'agit pas seulement d'estimations mais de coûts réels associés au diabète dans le système de santé. L'utilisation, comme paramètre, des coûts imputables au diabète est également judicieuse, car il s'agit de coûts supérieurs aux dépenses moyennes en santé, qui ont été obtenus par comparaison avec le groupe non diabétique. L'utilisation des coûts fondés uniquement sur la population malade peut donner lieu à une surestimation des dépenses relatives à la maladie et fournir des évaluations gonflées ${ }^{2}$.

Deuxièmement, cette méthode est simple d'application et peut être utilisée par de nombreux utilisateurs finaux. C'est ce qu'on vise avec cet outil : qu'il soit accessible et facile d'utilisation au sein de différents contextes d'application, comme les ministères de la santé provinciaux ou les instances régionales de la santé. Être en mesure de modéliser les scénarios d'intervention en fonction des objectifs du programme de l'utilisateur et des caractéristiques de la région est un autre avantage pour les responsables de la planification des services de santé et pour les décideurs qui souhaitent pouvoir estimer le contrepoids économique constitué par les diverses stratégies de prévention du diabète. Pouvoir estimer les coûts évités et le nombre de cas prévenus au moyen de stratégies d'intervention bien adaptées permet d'évaluer les différentes options politiques et peut contribuer à façonner les choix en matière d'activités de prévention des maladies chroniques. Par exemple, au Canada, il existe des douzaines de possibilités de politiques et d'interventions prometteuses visant à favoriser un mode de vie sain menées au moyen de partenariats fédéraux, provinciaux et régionaux ${ }^{18}$. De tels programmes tireraient avantage d'un outil qui tiendrait compte de facteurs comme les caractéristiques d'une population dans un contexte particulier pour déterminer les stratégies d'intervention les plus appropriées 
et les plus réalistes du point de vue de l'économie et de la santé. On pourrait inclure plus tard des données sur les résultats d'un meilleur traitement et diverses stratégies de prise en charge de la maladie. Comme ces approches peuvent prolonger la vie et peut-être réduire les coûts, ces données, combinées à l'effet sur l'incidence, permettraient d'avoir un aperçu de la combinaison de diverses approches en matière de traitement et en matière de prévention.

La simplicité de ce modèle implique que l'on émette et admette plusieurs hypothèses. D'abord, les estimations de coûts sont tirées d'une étude reposant sur les données obtenues en Ontario, ce qui fait que les coûts utilisés pour les estimations nationales reposent sur l'hypothèse que les dépenses en santé sont similaires dans les autres provinces et territoires. Il existe cependant des différences entre régions, en ce qui a trait notamment aux soins généraux et à la prise en charge du diabète $^{17}$ ainsi qu'à la couverture des services et des produits (comme les médicaments et les dispositifs ${ }^{19,20}$ de contrôle) offerte par les régimes provinciaux. Si d'autres estimations des coûts par province étaient disponibles à l'avenir, la méthode de calcul des coûts pourrait facilement être adaptée de façon à tenir compte des coûts spécifiques à chaque région.

Deuxièmement, cette méthode utilise des coûts imputables au diabète en fonction du sexe et de l'année de suivi, ce qui fait qu'elle ne peut pas prendre en compte les coûts évités au sein de certains sousgroupes susceptibles d'avoir soit davantage soit moins recours aux services de santé que la moyenne. Par exemple, dans le cadre d'une intervention visant le groupe à haut risque, il est probable que les personnes qui en font partie utilisent davantage de ressources en soins de santé que la moyenne, mais le calcul des coûts évités n'en tiendra pas compte (c.-à-d. qu'ils seront sous-estimés avec cette méthode). Réussir à obtenir des estimations de coûts pour des populations spécifiques permettrait des estimations plus précises, en particulier lors de la modélisation de scénarios d'intervention ciblant certains groupes-cibles.

Troisièmement, le modèle ne tient pas compte de changements à venir dans les dépenses en matière de santé ni de l'inflation. On suppose que la prise en charge des cas de diabète restera la même jusqu'en 2022 et que les modèles de soins actuels continueront à être appliqués et utilisés de la même façon. Sur 10 ans, cette hypothèse se justifie probablement, mais sur des périodes de prédiction plus longues on devrait tenir compte de changements potentiels en matière de soins et de prise en charge.

Enfin, nos estimations ne tiennent pas compte des coûts associés au diabète mais non liés aux soins de santé, que ce soit les coûts indirects, les frais non remboursables ou les coûts non saisis dans les bases de données administratives ou encore le coût émotionnel et social pour les patients et les autres fournisseurs de soins. Les coûts directement affectés aux soins de santé ne comptant selon les estimations que pour $17 \%$ des coûts totaux attribuables au diabète $^{3}$, ces dépenses supplémentaires devront être prises en compte dans les recherches à venir.

\section{Conclusion}

L'objectif de cette étude a été de fournir aux décideurs un outil convivial leur permettant d'obtenir des projections de coûts jusqu'à 10 ans. Les responsables de la planification en santé et de l'élaboration de politiques ciblant la prévention du diabète au sein de la population peuvent utiliser cet outil pour évaluer différentes stratégies d'intervention, en entrant une incidence et des prédictions de coûts particulières, ce qui les aidera à choisir pour l'avenir les mesures les plus appropriées.

\section{Remerciements}

$\mathrm{D}^{\text {re }}$ Laura Rosella est titulaire d'une chaire de recherche du Canada en analyse de la santé des populations. Ses travaux sont soutenus par une subvention de fonctionnement des Instituts de recherche en santé du Canada, décernée par l'Institut de la nutrition, du métabolisme et du diabète (financement $n^{\circ}$ 126615). Les bailleurs de fonds n'ont pas joué de rôle direct dans l'analyse, l'interprétation, la rédaction ou la présentation de ce manuscrit.

\section{Conflits d'intérêts}

Les auteurs n'ont aucun conflit d'intérêts à déclarer.

\section{Contribution des auteurs}

LR a conçu le manuscrit. LR et $A B$ ont toutes deux contribué au plan d'analyse et à l'interprétation. $\mathrm{AB}$ a préparé les données et effectué toutes les analyses. $\mathrm{AB}$ a rédigé l'ébauche du manuscrit et $L R$ a révisé et effectué une lecture critique du contenu final.

\section{Références}

1. Statistique Canada. Base de données CANSIM : Tableau 105-0501: Diabète, selon le groupe d'âge et le sexe [Internet]. Ottawa (Ont.) : Statistique Canada; 2014 [consulté le $1^{\text {er }}$ mai 2015]. En ligne à : http://www5 .statcan.gc.ca/cansim/a26?lang = fra \&id $=1050501$

2. Agence de la santé publique du Canada. Le fardeau économique de la maladie au Canada, 2005-2008, Ottawa (Ont.) : Agence de la santé publique du Canada; 2014. [nº HP50-1/2013F-PDF]

3. Somerville R. Un tsunami économique : le coût du diabète au Canada. Toronto (Ont.) : Association canadienne du diabète; 2009.

4. Goeree R, Lim ME, Hopkins R, et al. Prevalence, total and excess costs of diabetes and related complications in Ontario, Canada. Can J Diabetes. 2009;33(1):35-45.

5. Rosella LC, Lebenbaum M, Fitzpatrick $\mathrm{T}$, et al. Impact of diabetes on healthcare costs in a population-based cohort: a cost analysis. Diabet Med. 2016; 33(3):395-403.

6. Ohinmaa A, Jacobs P, Simpson S, Johnson JA. The projection of prevalence and cost of diabetes in Canada: 2000 to 2016. Can J Diabetes. 2004; 28(2):1-8.

7. Rosella LC, Manuel DG, Burchill C, Stukel TA, équipe PHIAT-DM. A population-based risk algorithm for the development of diabetes: development and validation of the Diabetes Population Risk Tool (DPoRT). J Epidemiol Community Health. 2011;65(7):613-20.

8. Rosella LC, Lebenbaum M, Li Y, Wang J, Manuel DG. Risk distribution and its influence on the population targets for diabetes prevention. Prev Med. 2014;58:17-21. 
9. Statistique Canada. Enquête sur la santé dans les collectivités canadiennes - Guide de l'utilisateur - Fichiers de microdonnées 2012 et 2011-2012. Ottawa (Ont.) : Statistique Canada; 2013

10. Comité d'experts des Lignes directrices de pratique clinique de l'Association canadienne du diabète. Lignes directrices de pratique clinique 2013 de l'Association canadienne du diabète pour la prévention et le traitement du diabète au Canada, Can J Diabetes, 2013;37 (suppl. 5):S3611-S598.

11. Glazier RH, Creatore MI, Weyman JT, et al. Density, destinations or both? A comparison of measures of walkability in relation to transportation behaviors, obesity and diabetes in Toronto, Canada. PLoS One. 2014;9(1):e85295. Erratum in PLoS One. 2014;9(3):e91485.

12. Gillies CL, Abrams KR, Lambert PC, et al. Pharmacological and lifestyle interventions to prevent or delay type 2 diabetes in people with impaired glucose tolerance: systematic review and meta-analysis. BMJ. 2007;334(7588): 299.

13. Wodchis WP, Bushmeneva K, Nikitovic M, McKillop I. Guidelines on person-level costing using administrative databases in Ontario. Toronto (ON) : Health System Performance Research Network; 2013.

14. De Melo M, de Sa E, Gucciardi E. Exploring differences in Canadian adult men and women with diabetes management: results from the Canadian Community Health Survey. BMC Public Health. 2013;13(1):1089.

15. Pohar SL, Majumdar SR, Johnson JA. Health care costs and mortality for Canadian urban and rural patients with diabetes: population-based trends from 1993-2001. Clin Ther. 2007;29 Spec No:1316-24.

16. Dawson KG, Gomes D, Gerstein H, Blanchard JF, Kahler KH. The economic cost of diabetes in Canada, 1998. Diabetes Care. 2002;25(8): 1303-1307.

17. Simpson SH, Corabian P, Jacobs P, Johnson JA. The cost of major comorbidity in people with diabetes mellitus. CMAJ. 2003;168(13):1661-1667.
18. Réseau pancanadien de santé publique. Vers un Canada plus sain - Rapport d'étape 2015 sur la promotion du cadre fédéral, provincial et territorial sur le poids santé [Internet]. En ligne à : http://www.phn-rsp.ca/thcpr-vcpsre -2015/index-fra.php

19. Association canadienne du diabète, Diabète Québec. Diabète : le Canada à l'heure de la remise en question : tracer une nouvelle voie, Toronto (Ont.) : Association canadienne du diabète et Diabète Québec; 2011.

20. Canadian Diabetes Association. The burden of out-of-pocket costs for Canadians with diabetes [Internet]. Toronto (Ont) : Canadian Diabetes Association [s.d.]. En ligne à : http:// www.diabetes.ca/CDA/media /documents / publications-and -newsletters/advocacy-reports/burden -of-out-of-pocket-costs-for-canadians -with-diabetes.pdf 